\title{
miR-122 inhibits viral replication and cell proliferation in hepatitis B virus-related hepatocellular carcinoma and targets NDRG3
}

\author{
CHUN-GUANG FAN $^{1 *}$, CHUN-MEI WANG ${ }^{1,2 *}$, CHUAN TIAN $^{3 *}$, YAN WANG $^{1}$, \\ $\mathrm{LI} \mathrm{LI}^{1}$, WEN-SHENG SUN ${ }^{4}$, RUI-FENG LI ${ }^{1}$ and YU-GANG LIU ${ }^{1}$ \\ Departments of ${ }^{1}$ Pathophysiology, ${ }^{2}$ Microbiology, Shandong University School of Medicine; \\ ${ }^{3}$ Department of Renal Transplant, Second Hospital of Shandong University; \\ ${ }^{4}$ Institute of Immunology, Shandong University School of Medicine, Jinan, P.R. China
}

Received May 12, 2011; Accepted June 17, 2011

DOI: 10.3892/or.2011.1375

\begin{abstract}
RNAs (miRNAs) are short, non-coding RNAs with post-transcriptional regulatory functions that participate in diverse biological pathways. miR-122, a liver-specific miRNA, has been found to be down-regulated in hepatocellular carcinoma (HCC) and HCC-derived cell lines. In this study, miR-122 was down-regulated in the hepatitis B virus (HBV)-related HCC cell line HepG2.2.15 compared to HepG2. NDRG3, a member of the N-myc downstream-regulated gene (NDRG) family, was up-regulated in HepG2.2.15 and was identified as a target gene of miR-122. An inverse correlation between the expression of miR-122 and the NDRG3 protein was noted in HBV-related HCC specimens. The transfection of the miR-122 expression vector into the HepG2.2.15 cell line repressed the transcription and expression of NDRG3, which subsequently reversed the malignant phenotype of the cells. The replication of $\mathrm{HBV}$, expression of viral antigens and proliferation of cells were significantly inhibited by restoration of miR-122. The data demonstrate that miR-122 plays an important role in HBV-related hepatocarcinogenesis by targeting NDRG3. Thus, miR-122 and NDRG3 represent key diagnostic markers and potential therapeutic targets for HBV-related HCC.
\end{abstract}

\section{Introduction}

Hepatocellular carcinoma (HCC) is one of the most aggressive human cancers worldwide and accounts for $80-90 \%$ of liver cancers $(1,2)$. Since the 1990 s, the rate of mortality associated

Correspondence to: Dr Yu-Gang Liu or Professor Rui-Feng Li, Department of Pathophysiology, Shandong University School of Medicine, 44 Wenhua Xi Road, Jinan 250012, P.R. China

E-mail: liu.yugang@sdu.edu.cn

E-mail: ruifeng@sdu.edu.cn

*Contributed equally

Key words: microRNAs, hepatitis B virus, hepatocellular carcinoma, miR-122, N-myc downstream-regulated gene 3 with HCC has remained the third highest worldwide and the second highest in China compared with all other cancers. The 5-year survival rate of HCC is less than 5\%; almost 600,000 patients die each year (3). Therefore, investigation into the pathogenesis of HCC is of great significance. The discovery of microRNAs (miRNAs), a class of small non-coding RNAs, identified a group of molecules that usually regulate target genes post-transcriptionally by binding to the complementary sequences (mostly in the 3'-UTR) of their target mRNAs and induce mRNA degradation or translational repression $(4,5)$. miRNAs are involved in the regulation of the growth and development of plants and animals as well as many other complicated life processes (6). The regulation of miRNAs also plays a key role in the occurrence of HCC (7). miR-122, a liver-specific miRNA, accounts for $70 \%$ of the miRNAs in the liver and is expressed at high levels in human and mouse liver, primary cultured liver cells and liver cell lines $(8,9)$. miR-122 impacts cell differentiation, proliferation, metabolism, stress and other liver processes by regulating the expression of a large number of target genes (10-12). Previous studies have shown that miR-122 is down-regulated in HCC and HCC-derived cell lines and contributes to hepatocarcinogenesis by targeting genes including cyclin G1, SRF, Igf1R, Bcl-w and ADAM17 (13-16). As a novel target for inhibiting the development of HCC, miR-122-based therapeutics may benefit the treatment of HCC, excluding hepatitis $\mathrm{C}$ virus infection (17).

Hepatitis B virus (HBV) infection induces hepatitis, cirrhosis and HCC, which are greatly detrimental to human health and affect quality of life (18). Therefore, it is of great importance to investigate the pathogenesis of HBV-related HCC. In our previous study, taking advantage of the fact that HBV-transgenic mice develop liver cancer spontaneously in the first 20 months of life (19), we observed the dynamic profiles of genes and miRNAs of HBV-transgenic mice as compared to syngeneic BALB/c mice. The expression level of miR-122 was significantly down-regulated in the HBV-transgenic mice, which was validated by real-time quantitative PCR. Levels of miR-122 were also significantly decreased when the cell line BEL7402 was transfected with the HBV expression plasmid, as well as in HepG2.2.15, as compared to HepG2. These data 
motivated us to further elucidate the relationship among HBV infection, miR-122 silencing and hepatocarcinogenesis. We report here that miR-122 targets NDRG3, a member of the $\mathrm{N}$-myc down-regulated gene (NDRG) family. Transfection of miR-122 into the HepG2.2.15 cell line reversed the malignant phenotype of cells and inhibited the replication of HBV and expression of viral antigens. Our data suggest an important role for miR-122 in HBV-related hepatocarcinogenesis by targeting NDRG3.

\section{Materials and methods}

Tissue samples and cell lines. Twenty-two pairs of tumor and adjacent non-tumor tissues were obtained from patients with HBV-related HCC at Shandong Provincial Hospital between 2008 and 2010. The tissue samples were snap-frozen in liquid nitrogen immediately after resection and stored at $-80^{\circ} \mathrm{C}$ until use. HCC cell lines (purchased from the American Type Culture Collection) were cultured using standard conditions for HepG2 in Dulbecco's modified Eagle's medium (DMEM; Hyclone, China) and $100 \mathrm{U} / \mathrm{ml}$ penicillin and streptomycin. HepG2.2.15 cells were cultured in Eagle's modified medium (MEM; Hyclone, China) and $380 \mathrm{mg} / \mathrm{l}$ antibiotic G-418 sulfate (Promega, USA), with 10\% fetal bovine serum (FBS; Gibco, USA) at $37^{\circ} \mathrm{C}$ in a $5 \% \mathrm{CO}_{2}$ incubator.

$R T$-PCR. Total RNA was isolated by TRIzol reagent(Invitrogen) according to the manufacturer's instructions. The first strand cDNA synthesis kit ReverTra Ace- $\alpha$ (Toyobo) was used for cDNA synthesis. PCR was performed in a single reaction using a 10- $\mu 1$ volume. Primers for the amplification of the NDRG3 gene were: forward, 5'-GAGATCACCCAGCACTTTGC-3', and reverse, 5'-AAGCTCCAGCTCCAACTCCA-3'. The human $\beta$-actin gene was used as an internal control and amplified with the following primers: forward, 5'-ACACTGT GCCCATCTACGAGGGG-3' and reverse, 5'-ATGATGGAG TTGAAGGTAGTTTCGTGGAT-3'.

PCR was conducted as follows: $94^{\circ} \mathrm{C}$ for $5 \mathrm{~min}$, followed by 30 cycles at $94^{\circ} \mathrm{C}$ for $30 \mathrm{sec}, 55^{\circ} \mathrm{C}$ for $30 \mathrm{sec}$ and $72^{\circ} \mathrm{C}$ for $30 \mathrm{sec}$. The PCR products were analyzed by electrophoresis on a $1 \%$ agarose gel.

qPCR. Real-time quantitative PCR was performed to detect the expression levels of miR-122 and NDRG3, respectively. Total RNA was extracted by TRIzol reagent according to the manufacturer's instructions.

For miR-122, an NCode VILO miRNA cDNA synthesis kit (Invitrogen) was used to obtain cDNA. An all-in-one miRNA qPCR primer set (GeneCopoeia) was used for miRNA-122 amplification. The level of U6 RNA was assayed to normalize the relative abundance of miR-122. PCR was performed using an ABI 7000 real-time PCR detector (ABI) with Express SYBR GreenER miRNA qRT-PCR kits (Invitrogen). After $50^{\circ} \mathrm{C}$ for $2 \mathrm{~min}, 95^{\circ} \mathrm{C}$ for $2 \mathrm{~min}, 40 \mathrm{ampli}-$ fication cycles were performed at $95^{\circ} \mathrm{C}$ for $15 \mathrm{sec}$ and $60^{\circ} \mathrm{C}$ for $60 \mathrm{sec}$. Fluorescence was recorded automatically during amplification, and the melting curve for the product was obtained.

For NDRG3, the ReverTra Ace qPCR RT kit (Toyobo) was used for reverse transcription. Primer Premier 5.0 was used to design primers for the NDRG3 gene: forward, 5'-GAGA TCACCCAGCACTTTGC-3' and reverse, 5'-AAGCTC CAGCTCCAACTCCA- 3 '. The human $\beta$-actin gene was measured as an internal control. qPCR was performed using SYBR Premix Ex Taq Perfect Real Time (Takara) according to the manufacturer's instructions. The cycling conditions were as followed: denaturation at $95^{\circ} \mathrm{C}$ for $30 \mathrm{sec}$, followed by 40 cycles of $95^{\circ} \mathrm{C}$ for $5 \mathrm{sec}, 60^{\circ} \mathrm{C}$ for $31 \mathrm{sec}$. To quantify the differences in NDRG3 mRNA levels in each group, the LightCycler data point curves were compared to similar analyses generated using a dilution standard.

Target prediction for miR-122. Computer-based programs including TargetScan (http://www.targetscan.org/), Pictar (http://pictar.bio.nyu.edu/) and MiRanda (http://microrna. sanger.ac.uk/) were used to predict potential targets for miR-122. We focused on NDRG3 as it appeared in the list of up-regulated genes in HBV transgenic mice as compared to $\mathrm{BALB} / \mathrm{c}$ mice. The duplex of miR-122 and the 3'-UTR of NDRG3 were predicted by RNAhybrid (http://bibiserv. techfak.uni-bielefeld.de/rnahybrid/).

miR-122 expression vector construction. According to the sequence of precursor miR-122 cDNA provided by GenBank, Primer Premier 5.0 was used to design the primers: forward, 5'-GTGACAATGGTGGAATGTGG-3' and reverse, 5'-AAA GCAAACGATGCCAAGAC-3'. The BLAST algorithm was used to prevent the designed sequences from targeting other gene transcripts. The restriction enzyme cutting site of BamHI (Takara Bio) was added to the 5' prime end of the forward primer; HindIII (Takara Bio) was added to the reverse primer and three protecting bases were added: forward, 5'-CTAG GATCCGTGACAATGGTGGAATGTGG-3' and reverse, 5'-CGCAAGCTTAAAGCAAACGATGCCAAGAC-3'. The primers were synthesized (BGI). Cell genomic DNA was extracted by a cell genomic DNA extraction kit (Solarbio) according to the manufacturer's instructions, and the target gene fragments were amplified. PCR products were collected, purified and digested by BamHI and HindIII and then ligated into pSilencer3.1-H1 neo digested with BamHI and HindIII. The product was denoted pS-miR122. Meanwhile, a control vector with non-sense sequences was constructed and denoted as pS-control. Vectors were propagated in DH5 $\alpha$ cells and then purified, digested and sequenced to ensure correct construction.

Cell transfection. Vector transfection was carried out with FuGENE 6 transfection reagent (Roche) according to the manufacturer's instructions. Cells were trypsinized and plated in 6-well plates at a density of $5.0 \times 10^{4}$ cells $/ \mathrm{ml}(2 \mathrm{ml} /$ well $)$ for $24 \mathrm{~h}$. Before transfection, culture medium was replaced with Opti-MEM without serum (Gibco). pS-miR122 and pS-control were transfected into the cells, with three wells for each group. The cells were replenished with fresh medium containing FBS and G-418 $6 \mathrm{~h}$ after transfection for further detection.

Western blot analysis. Seventy-two hours after transfection, cells were collected and analyzed by Western blot analysis to assess NDRG3 expression. Cells were lysed with $1 \%$ RIPA lysis buffer (Beyotime) in the presence of protease inhibi- 


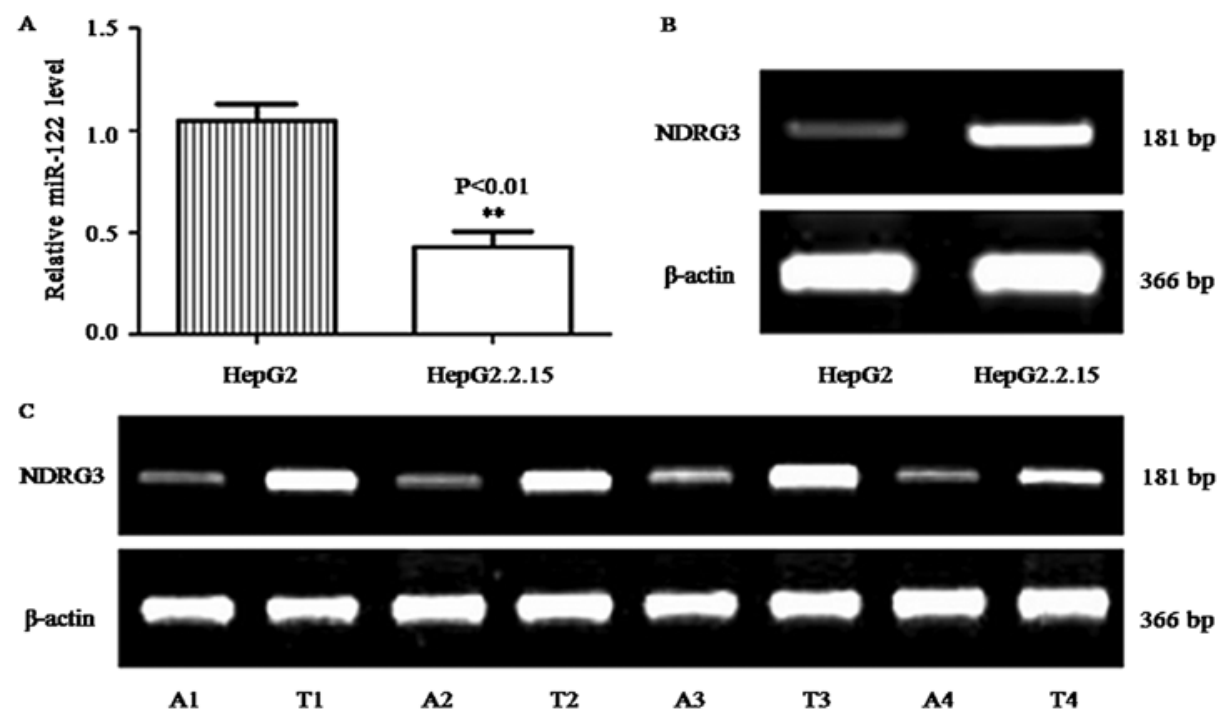

Figure 1. The expression levels of miR-122 and NDRG3 are inversely correlated in HBV-related HCC. (A) Real-time quantitative PCR was performed to detect the expression level of miR-122. The result shows that miR-122 is down-regulated in the HepG2.2.15 as compared to the HepG2 cell line (P<0.01). (B) The RT-PCR results show that NDRG3 is significantly up-regulated in HepG2.2.15 compared to HepG2 cells. (C) The RT-PCR results also show that NDRG3 is up-regulated in the HBV-related HCC tumor tissues (T1-T4), as compared to the adjacent non-tumor tissues (A1-A4) $(\mathrm{P}<0.01)$.

tors. Next, $20 \mathrm{mg}$ of total protein was detected by Bio-Rad protein assay, and $30 \mathrm{mg}$ of each sample was separated by sodium dodecyl sulfate-polyacrylamide gel electrophoresis and then transferred onto a PVDF membrane (Bio-Rad). After being blocked for $2 \mathrm{~h}$ at room temperature with a solution of phosphate-buffered saline and $0.05 \%$ Tween-20 containing $3 \%$ bovine serum albumin (BSA), the membranes were probed with rabbit polyclonal antibodies (Abcam) against human NDRG3 protein and then with horseradish peroxidase-conjugated secondary antibodies against rabbit (Abcam), followed by visualization with ECL.

HBsAg and HBeAg assay. The viral proteins hepatitis B surface antigen (HBsAg) and e antigen (HBeAg) of the supernatant from the transfected cells were quantified using an ELISA kit (Autbio, Zhengzhou, China) according to the manufacturer's instructions.

Detection of $H B V$-DNA by real-time PCR. Real-time PCR was performed to detect the HBV-DNA level using an HBV PCR fluorescence quantitative detection kit (Bioer) in the supernatant $48 \mathrm{~h}$ after transfection. Next, $100 \mu \mathrm{l}$ of the supernatant was collected according to the manufacturer's instructions. The DNA was extracted for real-time PCR using an ABI 7000 real-time PCR detector (ABI). Then, $2 \mu 1$ of DNA sample was added to the mixture of $37.7 \mu 1 \mathrm{HBV}$ PCR reaction solution, $0.3 \mu 1$ Taq enzyme and $0.1 \mu \mathrm{l}$ UDG enzyme. The PCR reaction program was as follows: $37^{\circ} \mathrm{C}$ for $5 \mathrm{~min}, 94^{\circ} \mathrm{C}$ for $2 \mathrm{~min}$, $95^{\circ} \mathrm{C}$ for $5 \mathrm{sec}, 60^{\circ} \mathrm{C}$ for $40 \mathrm{sec}, 40$ cycles. The fluorescence signal was collected at $60^{\circ} \mathrm{C}$. The standard curve for each test was obtained, and the correlation coefficient was $>0.99$. The experiment was repeated three times.

Cell viability assay. Cell-Counting Kit 8 (CCK8) (Beyotime) was used to observe cell proliferation after transfection, strictly following the manufacturer's instructions. Cells were cultured in 96-well plates; 0, 24, 48, and $72 \mathrm{~h}$ post-transfection, samples were assessed by the Bio-Rad protein assay.

Statistics. All experiments were performed in triplicate. The data were statistically evaluated by analysis of variance (ANOVA) and comparison of means (Student's t-test) at a significance level of $\mathrm{P}<0.05$. Both analyses were carried out using SPSS 12.0 statistical software.

\section{Results}

The expression of miR-122 and NDRG3 in HBV-related HCC is inversely correlated. The real-time qPCR assay showed that miR-122 was down-regulated in the HepG2.2.15 cells as compared to the HepG2 cells (Fig. 1A), which was in accordance with the results of miRNA chips in BEL7402-1.1HBV and the control BEL7402 cell line. The RT-PCR results showed that NDRG3 was up-regulated in the HepG2.2.15 compared to the HepG2 cells (Fig. 1B), which was consistent with the results of the DNA microarray of the HBV transgenic mice and control $\mathrm{BALB} / \mathrm{c}$ mice.

To ensure that this finding is significant in clinical patients, we demonstrated that NDRG3 was up-regulated in HBV-related hepatocellular carcinoma tissues as compared to the adjacent non-tumor tissues, as determined by RT-PCR (Fig. 1C).

NDRG3 is a candidate target gene of miR-122. By querying MiRanda and TargetScan, NDRG3 was identified in both programs as a target gene of miR-122. The prediction results and scores are shown in Fig. 2A. The TargetScan also showed the conserved binding sites on the 3'-UTR of NDRG3 across vertebrate species including the human, chimpanzee, Rhesus and the mouse (Fig. 2B). This indicates that miR-122 targeting to NDRG3 may be evolutionarily conserved. The free energy of miR-122 binding to the NDRG3 targeting site 
A

\begin{tabular}{|c|c|c|c|c|}
\hline \multicolumn{2}{|c|}{ predicted consequential pairing of target region (lop) and miRNA (bottom) } & $\begin{array}{l}\text { Sood } \\
\text { match }\end{array}$ & $\begin{array}{l}\text { TargetScan } \\
\text { context score }\end{array}$ & $\begin{array}{c}\text { MiRanda } \\
\text { score }\end{array}$ \\
\hline $\begin{array}{c}\text { Position } 47.53 \text { of NORG3 } 3 \text { ' UTR } \\
\text { Has-miR-122 }\end{array}$ & 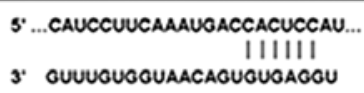 & 7 mer-1A & .0 .20 & 16.33 \\
\hline
\end{tabular}

B Hsa CAUUgCAAGUCCAUCCUUC....-AAAUGACCACUC-CAUAAUAUAA

Ptr CAUUGCAAGUCCAUCCUUC....-AAAUGACCACUC-CAUAAUAUAA

$\mathrm{Mml}$ AUUGCAAGUCCAUCCUUCUUCAAAUGACCACUC-CAUAAUAUAA

Mmu CAU.GCAAGUCCCUC.UCCUUGAAAUGACCACUC-.CGUAAUAGAA

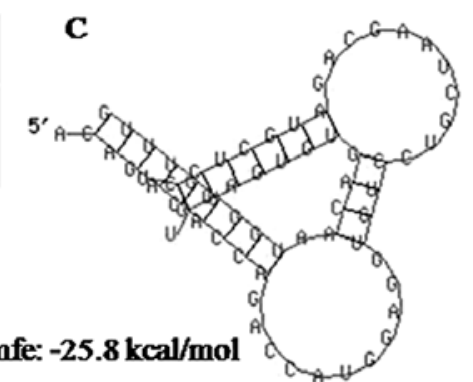

mfe: $-25.8 \mathrm{kcal} / \mathrm{mol}$

Figure 2. NDRG3 is a candidate target of miR-122. (A) By querying MiRanda and TargetScan, NDRG3 was identified in both programs as a potential target gene of miR-122. The prediction results and score are listed. (B) The TargetScan prediction shows a common binding sequence on the 3'-UTR of NDRG3 conserved across vertebrate species, including the human, chimpanzee, Rhesus and mouse. (C) The duplex of miR-122 and the 3'-UTR of NDRG3 are shown The free energy of miR-122 binding to the targeting site of NDRG3 was calculated using RNAhybrid (mfe, $-25.8 \mathrm{kcal} / \mathrm{mol}$ ).

A

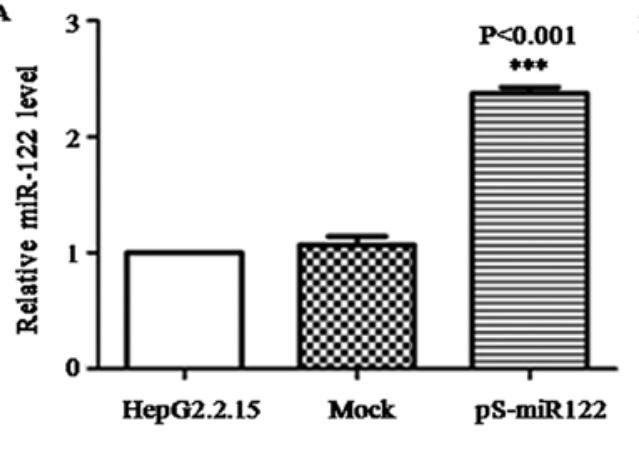

C
$\mathbf{B}$

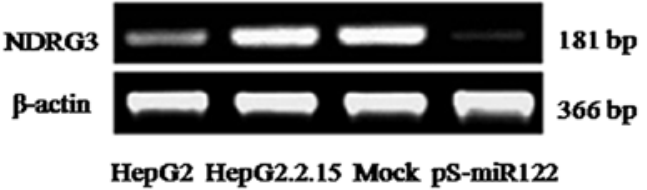

HepG2 HepG2.2.15 Mock pS-miR122

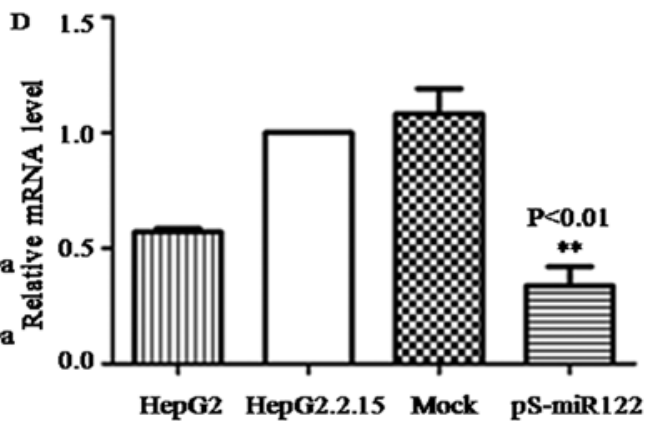

Figure 3. The expression level of NDRG3 is down-regulated by transfection with pS-miR122. (A) pEGFP-N1 was co-transfected with pS-miR122 into HepG2.2.15 cells. Compared to the mock and untreated cells, the expression level of miR-122 $48 \mathrm{~h}$ after pS-miR122 transfection was elevated in the HepG2.2.15 cells $(\mathrm{P}<0.001)$. (B) RT-PCR was performed $48 \mathrm{~h}$ post-transfection. mRNA of NDRG3 in cells transfected with pS-miR122 was significantly reduced compared to the mock and untreated cells $(\mathrm{P}<0.01)$. (C) The results of Western blot analysis show that the protein expression level of NDRG3 was down-regulated $72 \mathrm{~h}$ after transfection with pS-miR122, as compared to the mock and untreated cells (P<0.01). (D) The level of NDRG3 mRNA was detected by real-time qPCR $48 \mathrm{~h}$ post-transfection. The transcription of NDRG3 in cells transfected with pS-miR122 was significantly decreased, as compared to the mock and untreated cells $(\mathrm{P}<0.01)$.

was calculated using RNAhybrid (mfe, $-25.8 \mathrm{kcal} / \mathrm{mol}$ ), and the duplex of miR-122 and the 3'-UTR of NDRG3 was identified (Fig. 2C).

The expression level of NDRG3 is down-regulated by transfection of $p S-m i R 122$. To observe the transfection efficiency of these vectors into HepG2.2.15, pEGFP-N1 was co-transfected with pS-miR122 into HepG2.2.15 cells in 3-wells of another 6-well plate. Forty-eight hours after transfection, the expression of Emerald green fluorescent protein (EmGFP) was detected under fluorescence microscopy. The transfection efficiency of these vectors into HepG2.2.15 cells was $45-55 \%$ in this study. Forty-eight hours after transfection, the relative amount of miR-122 was measured by real-time quantitative PCR analysis.
Compared with mock and untreated cells, the expression level of miR-122 was elevated by transfection of pS-miR122 in the cells (Fig. 3A).

The mRNA expression level of NDRG3 was detected by RT-PCR and qPCR 48 h post-transfection. The result (Fig. 3B and D) showed that the mRNA expression levels of NDRG3 in cells transfected with pS-miR122 were significantly reduced as compared to the mock and untreated cells. The NDRG3 protein level was determined by Western blot analysis $72 \mathrm{~h}$ post-transfection (Fig. 3C), demonstrating that NDRG3 protein was significantly reduced in cells transfected with pS-miR122, as compared to the mock and untreated cells. These data suggest that NDRG3 is one of the direct downstream targets regulated by miR-122. 
A

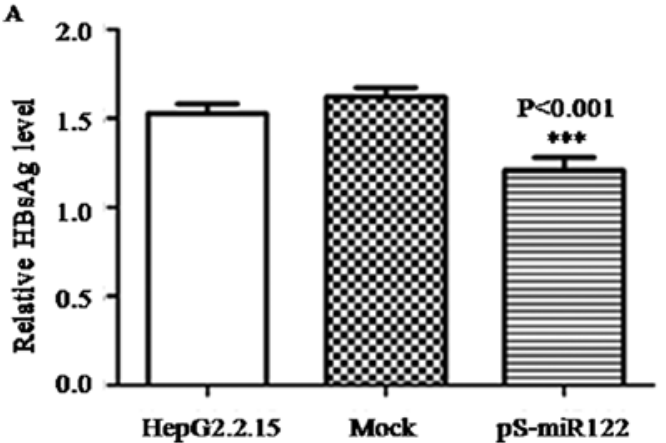

c

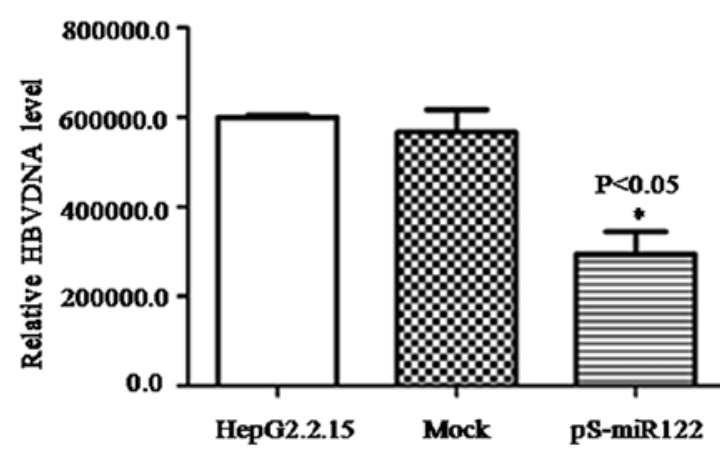

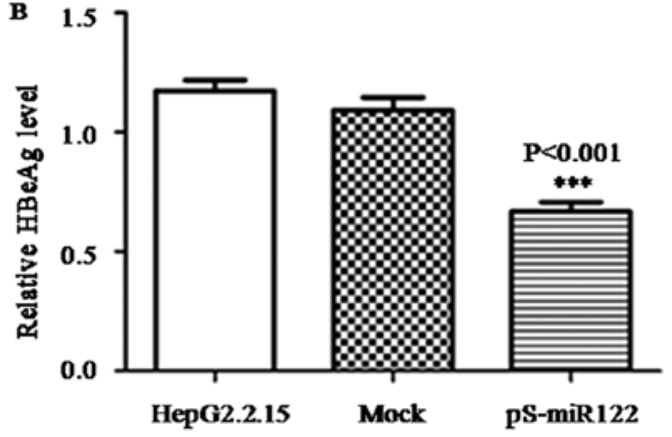

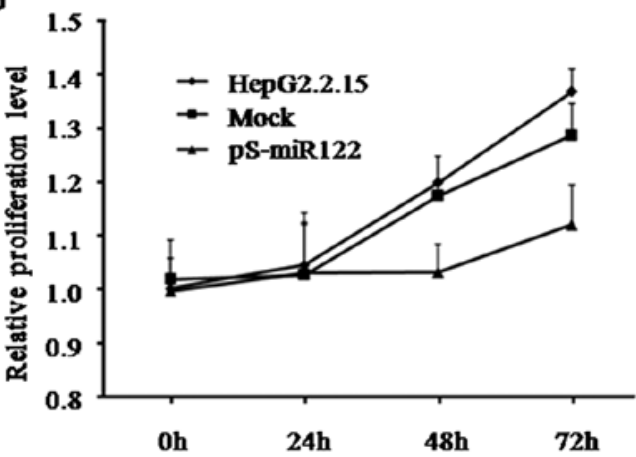

Figure 4. Transfection of pS-miR122 inhibits HBV replication and the proliferation of HepG2.2.15 cells. (A and B) Transfection with pS-miR122 had significant inhibitory effects on HBsAg and HBeAg as compared to the pS-control group $(\mathrm{P}<0.001)$. No obvious change was detected in the cells transfected with PS-control (P>0.05). (C) The HBV-DNA level was inhibited by transfection with pS-miR122. Real-time fluorescence quantitative PCR was used to detect HBV-DNA levels of cells $48 \mathrm{~h}$ post-transfection with pS-miR122. The copies of HBV-DNA were significantly decreased compared with the pS-control group (P<0.05). (D) Proliferation of cells transfected with pS-miR122 was obviously inhibited as compared with pS-control transfection 48 and $72 \mathrm{~h}$ post-transfection.

HBeAg and HBsAg secretion and HBV DNA replication are inhibited by transfection of $p S$-miR122. Forty-eight hours after transfection, the concentrations of HBsAg and HBeAg in the supernatant were detected using ELISA kits. Transfection of pS-miR122 had significant inhibitory effects on the expression of $\mathrm{HBsAg}$ and $\mathrm{HBeAg}$ (Fig. 4A and B), as compared to the control group. Real-time fluorescence quantitative PCR was performed to determine the HBV DNA level. The results showed that HBV DNA replication of cells transfected with pS-miR122 was reduced $48 \mathrm{~h}$ after transfection (Fig. 4C). No obvious change was observed in the HepG2.2.15 cells transfected with $\mathrm{pS}$-control when compared to the non-transfected cells $(\mathrm{P}>0.05)$.

Expression of miR-122 inhibits cell proliferation. Proliferation of cells transfected with pS-miR122 was obviously inhibited as compared to the $\mathrm{pS}$-control at 48 and $72 \mathrm{~h}$ post-transfection (Fig. 4D). These data confirmed that NDRG3 down-regulation as a target of miR-122 significantly inhibited the proliferation of HepG2.2.15 cells. Thus, miR-122 plays an important role in HBV-related HCC by targeting NDRG3.

\section{Discussion}

The most abundant microRNA in the liver is miR-122, which has been investigated extensively since 2002 to demonstrate its biological functions and significance in various diseases $(9,20)$. The down-regulation or loss of miR-122 has been found to contribute to hepatocarcinogenesis, suggesting that miR-122 acts as a tumor-suppressor miRNA $(14,16,21)$. In the present study, we showed that miR-122 was down-regulated in HBV-related HCC and targets NDRG3, suggesting that miR-122 plays an important role in HBV-related hepatocarcinogenesis. The transfection of the miR-122 expression vector into the HepG2.2.15 cell line repressed the transcription and expression of NDRG3. Furthermore, the replication of HBV and the expression of viral antigens, as well as proliferation of cells, were significantly inhibited by the restoration of miR-122 and suppression of NDRG3.

The NDRG family was discovered in 2002 and contains 4 paralogs: NDRG1, 2, 3, and 4 (22). The NDRG family shares a conserved Ndr domain. NDRG3 is expressed in many organs, with the highest expression levels confirmed in the testis and prostate of mice (23). It appears to be important in mouse spermatogenesis, but the mechanism remains unknown. NDRG3 was found to be a tumor promoter and its overexpression may contribute to the malignant phenotype of prostate cancer (24). However, the function of NDRG3 and its relationship to other cancer types remains unknown. We found that NDRG3 was up-regulated in HBV-transgenic mice during hepatocarcinogenesis, similar to the HBV carrier state (unpublished data). In the present study, we showed that NDRG3 was overexpressed in both clinical tumor specimens of HBV-related HCC and the HepG2.2.15 cell line expressing HBV antigens. This finding suggests that NDRG3 may play a role in HBV-related HCC and may be a candidate molecular diagnostic biomarker and a potential therapeutic target for further investigations. The inversely correlated expression of miR-122 and NDRG3 in HBV-related HCC motivated us to study the mechanisms in detail. By querying MiRanda 
and TargetScan, NDRG3 was identified in both programs as a target gene of miR-122. Overexpression of miR-122 in HepG2.2.15 cells silenced NDRG3 transcription and expression, which shows that NDRG3 is a novel target regulated by miR-122. The mechanisms by which miR-122 inhibits NDRG3 should be investigated in further research, including the polymorphism at the miR-122 binding site in the 3 ' untranslated region of NDRG3. The relationship between HBV infection and miR-122 down-regulation should also be investigated by elucidating the functional polymorphism in the promoter region of miR-122, microRNA DNA methylation, and the site at which HBV inserts into the human genome. Future studies will elucidate the molecular pathogenesis during HBV infection and hepatocarcinogenesis.

Several target genes of miR-122 have been identified in recent reports (13-16). The data imply that miR-122 could serves as a potential therapeutic target. PCR-generated linear DNA expression cassettes, which are free of antibiotic resistance genes typically found in plasmid DNA, were used to silence HBV replication in cell culture and in vivo (25). The method may be helpful for the development of safe and efficient anti-HBV gene therapy. We are currently evaluating the effect of miR-122 transfection in vivo using a mouse model induced by hydrodynamic-based tail vein injection, which was used in a previous study for the liver protection of soluble Death Receptor 5 (26). Consistent in vitro and in vivo data may build the basis for future clinical practice. However, miR-122 may be not suitable for patients with infection and HCC induced by hepatitis $\mathrm{C}$ virus since the sequestration of miR-122 in liver cells results in a marked loss of autonomously replicating hepatitis C viral RNAs (27). Therefore, miR122-based therapeutics might be a double-edged sword in the treatment of HCC. If such an approach is applied, the status of HCV infection in patients should be carefully assessed (17).

In conclusion, we found that during the process of HBV infection and HCC occurrence, the suppression of miR-122 up-regulates the corresponding target gene, NDRG3. The restoration of miR-122 expression effectively represses the transcription and expression of NDRG3, as well as inhibits the replication of HBV and the proliferation of cells. Further study should be carried out to aid in the development of a new strategy to prevent the chronicity of HBV infection and the development of HCC. Such theoretical and experimental support may improve the quality of clinical prevention and treatment.

\section{Acknowledgements}

We are grateful to Professor Youhai H. Chen, Department of Pathology and Laboratory Medicine, University of Pennsylvania, for kindly providing the pSilencer3.1-H1 neo. This study was supported by the National Natural Science Foundation of China (grant no. 30801036 to Y.-G. Liu and no. 30772031 for W.-S. Sun), as well as the Doctorate Fund for New Teachers from the National Education Ministry of China (grant no. 200804221065 for Y.-G. Liu).

\section{References}

1. Seeff LB and Hoofnagle JH: Epidemiology of hepatocellular carcinoma in areas of low hepatitis B and hepatitis C endemicity. Oncogene 25: 3771-3777, 2006.
2. Thorgeirsson SS and Grisham JW: Molecular pathogenesis of human hepatocellular carcinoma. Nat Genet 31: 339-346, 2002.

3. Parkin DM, Bray F, Ferlay $\mathbf{J}$ and Pisani P: Global cancer statistics, 2002. CA Cancer J Clin 55: 74-108, 2005.

4. Ambros V: The functions of animal microR NAs. Nature 431: 350-355, 2004.

5. Meister G and Tuschl T: Mechanisms of gene silencing by double-stranded RNA. Nature 431: 343-349, 2004.

6. Bartel DP: MicroRNAs: genomics, biogenesis, mechanism, and function. Cell 116: 281-297, 2004.

7. Skaftnesmo KO, Prestegarden L, Micklem DR and Lorens JB MicroRNAs in tumorigenesis. Curr Pharm Biotechnol 8: 320-325, 2007.

8. Lagos-Quintana M, Rauhut R, Lendeckel W and Tuschl T: Identification of novel genes coding for small expressed RNAs. Science 294: 853-858, 2001

9. Lagos-Quintana M, Rauhut R, Yalcin A, Meyer J, Lendeckel W and Tuschl T: Identification of tissue-specific microRNAs from mouse. Curr Biol 12: 735-739, 2002.

10. Lim LP, Lau NC, Garrett-Engele P, et al: Microarray analysis shows that some microRNAs downregulate large numbers of target mRNAs. Nature 433: 769-773, 2005.

11. Esau C, Davis S, Murray SF, et al: miR-122 regulation of lipid metabolism revealed by in vivo antisense targeting. Cell Metab 3: 87-98, 2006.

12. Bhattacharyya SN, Habermacher R, Martine U, Closs EI and Filipowicz W: Relief of microRNA-mediated translational repression in human cells subjected to stress. Cell 125: 1111-1124, 2006.

13. Gramantieri L, Ferracin M, Fornari F, et al: Cyclin G1 is a target of miR-122a, a microRNA frequently down-regulated in human hepatocellular carcinoma. Cancer Res 67: 6092-6099, 2007.

14. Bai S, Nasser MW, Wang B, et al: MicroRNA-122 inhibits tumorigenic properties of hepatocellular carcinoma cells and sensitizes these cells to sorafenib. J Biol Chem 284: 32015-32027, 2009.

15. Lin CJ, Gong HY, Tseng HC, Wang WL and Wu JL: miR-122 targets an anti-apoptotic gene, Bcl-w, in human hepatocellular carcinoma cell lines. Biochem Biophys Res Commun 375: 315-320, 2008.

16. Tsai WC, Hsu PW, Lai TC, et al: MicroRNA-122, a tumor suppressor microRNA that regulates intrahepatic metastasis of hepatocellular carcinoma. Hepatology 49: 1571-1582, 2009.

17. Zhang R, Wang L, Yu GR, Zhang X, Yao LB and Yang AG: MicroRNA-122 might be a double-edged sword in hepatocellular carcinoma. Hepatology 50: 1322-1323, 2009.

18. Lavanchy D: Hepatitis B virus epidemiology, disease burden, treatment, and current and emerging prevention and control measures. J Viral Hepat 11: 97-107, 2004.

19. Barone M, Maiorano E, Ladisa R, et al: Influence of ursodeoxycholate-enriched diet on liver tumor growth in HBV transgenic mice. Hepatology 37: 880-886, 2003.

20. Girard M, Jacquemin E, Munnich A, Lyonnet S and HenrionCaude A: miR-122, a paradigm for the role of microRNAs in the liver. J Hepatol 48: 648-656, 2008.

21. Fornari F, Gramantieri L, Giovannini C, et al: MiR-122/cyclin G1 interaction modulates p53 activity and affects doxorubicin sensitivity of human hepatocarcinoma cells. Cancer Res 69: 5761-5767, 2009.

22. Qu X, Zhai Y, Wei H, Zhang C, Xing G, Yu Y and He F: Characterization and expression of three novel differentiationrelated genes belong to the human NDRG gene family. Mol Cell Biochem 229: 35-44, 2002.

23. Zhao W, Tang R, Huang Y, et al: Cloning and expression pattern of the human NDRG3 gene. Biochim Biophys Acta 1519: 134-138, 2001.

24. Wang W, Li Y, Li Y, Hong A, Wang J, Lin B and Li R: NDRG3 is an androgen-regulated and prostate-enriched gene that promotes in vitro and in vivo prostate cancer cell growth. Int J Cancer 124: 521-530, 2009.

25. Chattopadhyay S, Ely A, Bloom K, Weinberg MS and Arbuthnot P: Inhibition of hepatitis B virus replication with linear DNA sequences expressing antiviral micro-RNA shuttles. Biochem Biophys Res Commun 389: 484-489, 2009.

26. Liu YG, Liu SX, Liang XH, et al: Blockade of TRAIL pathway ameliorates HBV-induced hepatocyte apoptosis in an acute hepatitis model. Biochem Biophys Res Commun 352: 329-334, 2007.

27. Jopling CL, Yi M, Lancaster AM, Lemon SM and Sarnow P: Modulation of hepatitis $\mathrm{C}$ virus RNA abundance by a liverspecific microRNA. Science 309: 1577-1581, 2005. 